\title{
Mgs1 function at G-quadruplex structures during DNA replication
}

\author{
Katrin Paeschke ${ }^{1} \cdot$ Peter Burkovics ${ }^{2}$ (1)
}

Received: 25 September 2020 / Revised: 27 October 2020 / Accepted: 29 October 2020 / Published online: 25 November 2020 (c) The Author(s) 2020

\begin{abstract}
The coordinated action of DNA polymerases and DNA helicases is essential at genomic sites that are hard to replicate. Among these are sites that harbour G-quadruplex DNA structures (G4). G4s are stable alternative DNA structures, which have been implicated to be involved in important cellular processes like the regulation of gene expression or telomere maintenance. G4 structures were shown to hinder replication fork progression and cause genomic deletions, mutations and recombination events. Many helicases unwind G4 structures and preserve genome stability, but a detailed understanding of G4 replication and the re-start of stalled replication forks around formed G4 structures is not clear, yet. In our recent study, we identified that Mgs 1 preferentially binds to G4 DNA structures in vitro and is associated with putative G4-forming chromosomal regions in vivo. Mgs1 binding to G4 motifs in vivo is partially dependent on the helicase Pif1. Pif1 is the major G4-unwinding helicase in S. cerevisiae. In the absence of Mgs1, we determined elevated gross chromosomal rearrangement (GCR) rates in yeast, similar to Pif1 deletion. Here, we highlight the recent findings and set these into context with a new mechanistic model. We propose that Mgs1's functions support DNA replication at G4-forming regions.
\end{abstract}

Keywords G-quadruplex $\cdot$ Replication $\cdot$ Mgs1 $\cdot$ Genome stability

\section{Introduction}

Precise replication of the genome is essential for most eukaryotic cells, as it determines the fate of the daughter cells. Failure of precise replication can lead to genome instability, cancerous transformation or apoptosis. The continuous movement of the replication fork is often stalled by various obstacles, like different hard-to-replicate alterations on the template DNA strand, DNA-bound protein complexes, DNA damage or stable secondary structures (Aguilera and GarciaMuse 2013). The stalled replication fork can be rescued by

Communicated by M. Kupiec.

Katrin Paeschke and Peter Burkovics contributed equally to this work.

Katrin Paeschke

kpaeschk@uni-bonn.de

$\triangle$ Peter Burkovics

burkovics.peter@brc.hu

1 Department of Oncology, Hematology and Rheumatology, University Hospital Bonn, Bonn, Germany

2 Institute of Genetics, Biological Research Centre, Szeged, Hungary different pathways, including a direct bypass of the lesion or template switching where the newly synthesised DNA strand serves as a template (Unk et al. 2010). Post-translational modifications of PCNA, a homotrimer ring-like protein, regulates the re-start/repair of the stalled fork (Moldovan et al. 2007; Arbel et al. 2020; Ripley et al. 2020). Ubiquitylation of PCNA by the Rad6/Rad18 complex activates the DNA damage tolerance pathway (Hoege et al. 2002) whereas PCNA SUMOylation inhibits unwanted recombination events at the stalled fork (Papouli et al. 2005; Pfander et al. 2005; Motegi et al. 2006; Burkovics et al. 2013).

DNA can adopt alternative secondary structures in addition to the standard B-DNA conformation. The G-quadruplex (G4) structure is a stable, alternative DNA or RNA structure, which can form in specific guanine-rich sequences. The core of this structure is a G-quartet: four guanines form a planar cyclic arrangement which is stabilized by Hoogsteen base pairing. Stacking of G-quartets leads to a higher ordered structure that is stabilized by monovalent cations, most frequently potassium (Lipps and Rhodes 2009; Bochman et al. 2012; Chen and Yang 2012). Genomic regions with a high potential to fold into G4 structures can be determined experimentally as well as computationally (Huppert and Balasubramanian 2007; Todd and Neidle 2011; 
Hansel-Hertsch et al. 2017; Marsico et al. 2019). G4s were identified at telomeres and many endogenous sites (intrachromosomally) in all eukaryotic cells tested so far. It is assumed that depending on the cell cycle, developmental phases, exogenous or endogenous stimuli different G4 structures form within the cell and mediate alternative events (Juranek and Paeschke 2012; Spiegel et al. 2020). Because of their high stability, the formation of G4 structures needs to be tightly regulated. Misregulated G4 structures or G4 structures formed at the wrong time or location can lead to genome instability. Different experimental approaches have shown that G4 formation can alter transcription, translation and the activity of polymerases and telomerase (Bochman et al. 2012; Rhodes and Lipps 2015; Muellner and Schmidt 2020; Varshney et al. 2020). In summary, misregulated G4 structures lead to a stalled or slowed DNA replication machinery and increase the number of chromosomal mutations, deletions and recombination events (Valton and Prioleau 2016; Bryan 2019; Lerner and Sale 2019). Based on these data, it would be expected that these sequences disappear during evolution. However, the situation is the opposite. During the evolution, the amount of potentially G4-forming sequences increased and the regions that could form G4 structures are more evolutionary conserved than neighbouring regions (Nakken et al. 2009; Capra et al. 2010; Marsico et al. 2019). This indicates a positive function of G4 structures at these regions, most likely in fine-tuning of cellular processes. To counteract the negative genome instability effects, but still benefit from the positive regulatory potential of G4 structures, cells must have developed machinery to control G4 structure formation.

\section{DNA helicases are needed for $\mathbf{G} 4$ replication}

In the past years, several different G4-unwinding helicases have been identified (Mendoza et al. 2016; Sauer and Paeschke 2017). They differ from each other based on their directionality as well as their processivity at G4 structures. It is interesting to note that although these helicases unwind G4 structures in vitro they are specific for only a certain set of G4 structures in vivo. It is not clear, yet, how they gain specificity for specific target G4 structures. In Saccharomyces cerevisiae at least three DNA helicases can unwind G4 structures in vitro (Pif1, Sgs1 and Hrq1) and have been implicated to function at G4 regions in vivo (Sun et al. 1999; Ribeyre et al. 2009; Piazza et al. 2010; Paeschke et al. 2011, 2013; Byrd and Raney 2015; Hou et al. 2015; Rogers et al. 2017; Dahan et al. 2018; Sparks et al. 2019). Pif1 seems to be the primary G4-unwinding helicase in yeast (Ribeyre et al. 2009; Paeschke et al. 2013). Pif1 is a highly conserved 5'-3' DNA helicase, which belongs to the SF1 superfamily (Bochman et al. 2010). Pif1 has a mitochondrial and a nuclear isoform (Foury and Dyck 1985; Schulz and Zakian 1994) and multiple functions in the cell. All of these functions are linked to the preservation of genome stability: (I.) Pif1 activity is essential for the maintenance of the mitochondrial genome (Foury and Dyck 1985), (II.) Pif1 cooperates with proteins of the replication machinery (Dna2 and PCNA) (Budd et al. 2006; Buzovetsky et al. 2017) and supports Okazaki-fragment maturation (Stith et al. 2008; Pike et al. 2009), (III.) Pif1 co-localizes with DNA repair foci and suppresses the accumulation of toxic DNA recombination intermediates (Wagner et al. 2006; Wilson et al. 2013), (IV.) Pif1 maintains the replication fork barrier at the ribosomal DNA loci, (Ivessa et al. 2000), (V.) Pif1 negatively regulates telomerase (Schulz and Zakian 1994; Boule et al. 2005; Phillips et al. 2015) and (VI.) Pif1 is associated with putative G4-forming regions in the yeast genome. Pif1 binds and unwinds G4 structures and supports DNA replication (Paeschke et al. 2011, 2013). The strand specificity of Pif1 is not clear yet, but most likely it can act on both leading and lagging strand template DNA (Lopes et al. 2011; Dahan et al. 2018). It is assumed that Pif1's function is supported by additional proteins. It has been shown that Mms1 supports Pif1-binding to a subset of G4 motifs located on the lagging strand template DNA at replication (Wanzek et al. 2017). Surprisingly, Pif1 can unwind G4 structures in an ATP-dependent and ATP-independent manner (Byrd et al. 2018). The current model of the mechanism of Pif1 function at G4 structures during replication is that Pif1 slides on the single-stranded template DNA in $5^{\prime}-3^{\prime}$ direction in an ATPdependent manner and resolves G4 structures as a monomer. After the unfolding of the G4 structure, Pif1 is stalled at the primer-template junction of the replication fork. At this point Pif1 is dimerising and the dimer can efficiently unwind the dsDNA after the junction point. Additionally, Pif1 can reanneal at the junction point the complementary strand of the G4-forming sequence via its strand-annealing activity, which could be a potential way to prevent the re-formation of the unfolded G4 structure (Galletto and Tomko 2013; Zhou et al. 2014; Duan et al. 2015; Li et al. 2016; Zhang et al. 2016).

\section{Mgs1 preserves genome stability at the replication fork}

S. cerevisiae Mgs1 (Maintenance of genome stability 1) is a multifunctional protein which belongs to the conserved AAA ${ }^{+}$ATPase family (Hishida et al. 2001). The exact biochemical mechanism of its action is not known, but its function in genome maintenance was clearly demonstrated: (I.) Its absence leads to an elevated rate of mitotic recombination (Hishida et al. 2001), (II.) overexpression of Mgs1 results in increased DNA damage sensitivity of yeast cells (UV, HU, and MMS) (Hishida et al. 2001, 2002; Branzei et al. 2002a, 
b) and (III.) $m g s 1$ is synthetically lethal with $\operatorname{rad} 6 \Delta$ and shows a synergistic growth defect with rad18 (Hishida et al. 2002). This data suggests a Rad18-independent, replicationassociated function of Mgs1. Mgs1 might be involved in the rescue of stalled replication forks (Barbour and Xiao 2003; Hishida et al. 2006; Vijeh Motlagh et al. 2006). Mgsl $\operatorname{sgsl\Delta }$ yeast cells show a slow-growing phenotype (Branzei et al. 2002a, b), Mgs1 stimulates the activity of the DNA polymerase $\delta$ (Branzei et al. 2002a, b) and Mgs1 is required to inhibit a recombination salvage pathway at stalled replication forks (Jimenez-Martin et al. 2020). Additionally, Mgs1 may also act in Okazaki-fragment maturation via stimulation of the Fen 1 endonuclease (Kim et al. 2005). Mgs1 has a UBZ domain, located at the $\mathrm{N}$-terminal part of the protein and an ATPase domain at the central region (Lehmann et al. 2020). Mgs1 exhibits a DNA-dependent ATPase and single-strand annealing activity (Hishida et al. 2001). These functions are connected to its ATPase domain. Mgs1 interacts with PCNA and exhibits a preference for the association with polyubiquitylated PCNA (Saugar et al. 2012).

The synthetic lethal phenotype of the $m g s 1 \Delta \operatorname{rad} 6 \Delta$ strain can be rescued by overexpression of Mgs1 lacking the UBZ domain (Saugar et al. 2012). This suggests that the Mgs1dependent rescue of the stalled fork is independent of the DNA damage tolerance pathway and PCNA ubiquitylation. We recently performed an in vivo yeast-one hybrid screen and identified novel G4 interacting proteins. We identified over 100 protein candidates including Slx9 and Zuo1 (Gotz et al. 2019; De Magis et al. 2020). Their G4-binding was already confirmed in vivo and in vivo. We also identified novel G4 structure-binding candidate proteins. The $\mathrm{Y} 1 \mathrm{H}$ has the advantage that the screen is done in vivo-protein folding and G4 formation are not altered because of purification steps or biochemical changes. Among these new proteins was Mgs1. It caught our interest due to its role in DNA replication. We confirmed that Mgs1 specifically binds G4 structures in vivo. The binding affinity to G4 structures was 3-to-10-fold higher compared to unstructured DNA. Although the binding affinity of Mgs1 was specific for G4 DNA, we could not monitor a change in ATPase activity upon G4 structure binding (Zacheja et al. 2020).

The binding specificity of Mgs1 to G4 structures in vitro was the first indication of a possible function at G4 structures also in vivo. It did not answer the questions if, when and why Mgs1 binds to G4 structures in vivo. Previous studies have shown that the timing of binding to G4 structures is particularly important. Slx 9 only binds to G4 structures during DNA damaging conditions, whereas Pif1 binds to G4 structures only during S phase (Paeschke et al. 2011; Gotz et al. 2019). Similarly to Pif1, Mgs1 binds to G4 structures in vivo even without the addition of DNA damage (Zacheja et al. 2020). The binding of Mgs1 is even stronger/enriched if G4 structures are stabilized by the G4-stabilizing ligand
PhenDC $_{3}$. The association of Mgs1 to the G4 structure depends on the presence of Pif1 but it is independent of Sgs1 (Zacheja et al. 2020). This data suggested that Pif1 and Mgs1 act in the same pathway because Pif1's function partially supports Mgs1-binding to G4 structures. We built a hypothetical model which describes the function of Mgs1 in the replication of G4 structures in association with Pif1 action, based on the available data. We demonstrated that Mgs1's function at G4 structures is essential for genome stability and that G4 structures that lack Mgs 1 (in $m g s 1 \Delta$ cells) caused increased GCR, accumulation of $\gamma \mathrm{H} 2 \mathrm{~A}$ as well as slow growth. We did not observe any alteration in replication fork progression in $m g s l \Delta$ cells under normal conditions.

The current model is that G4 structures, which form during DNA replication, lead to a slowing down of the replication fork as it approaches the G4 structure (Paeschke et al. 2011). Pif1 is recruited and unwinds these G4 structures and suppresses genome instability at these sites (Paeschke et al. 2011). Another work has shown that G4 structures are unfolded or repaired in the next cell cycle (Lemmens et al. 2015). Our data shows that without Mgs 1 more DNA damage and genomic rearrangement is observed at G4 structures (Zacheja et al. 2020). We predict that Mgs1 functions to protect the slowed/stalled replication fork at the G4 structures. The major question is how Pif1 binding is connected to Mgs1 binding at G4 structures. We did not observe any direct interaction of Pif1 and Mgs1, but assume that they are functionally connected. Based on published data we predict the following model (Fig. 1): (I.) G4 structures induce stalling of the replication fork, (II.) Mgs1 protects stalled replication forks and anchors them to the G4 structure, (III.) Pif1 resolves G4 structures and interacts with the replication complex via its interaction with PCNA. The unwinding of G4 structures and the re-start of the stalled replication fork stabilizes Mgs1 in the replication complex in concert with Pif1 binding. At this point, we cannot exclude that in the absence of PCNA protein, which is a binding partner of Mgs1 and Pif1, is modified or altered in such a manner that Mgs1 binding is reduced. In summary, we predict that Mgs1 is recruited to G4 structures formed during DNA replication and that its major function is to protect the replication fork and prevent genome instability.

\section{Further perspectives}

How are the intrachromosomal G4 structure-forming sequences replicated? Several questions are still unanswered regarding this process. One of the main questions is the timing of G4-unwinding in wild type cells. Can the normal replication apparatus handle this situation or do all formed G4 structures lead to replication fork stalling? If stalling of the replication fork is induced at every formed 
Replication stalls at the G4 structure
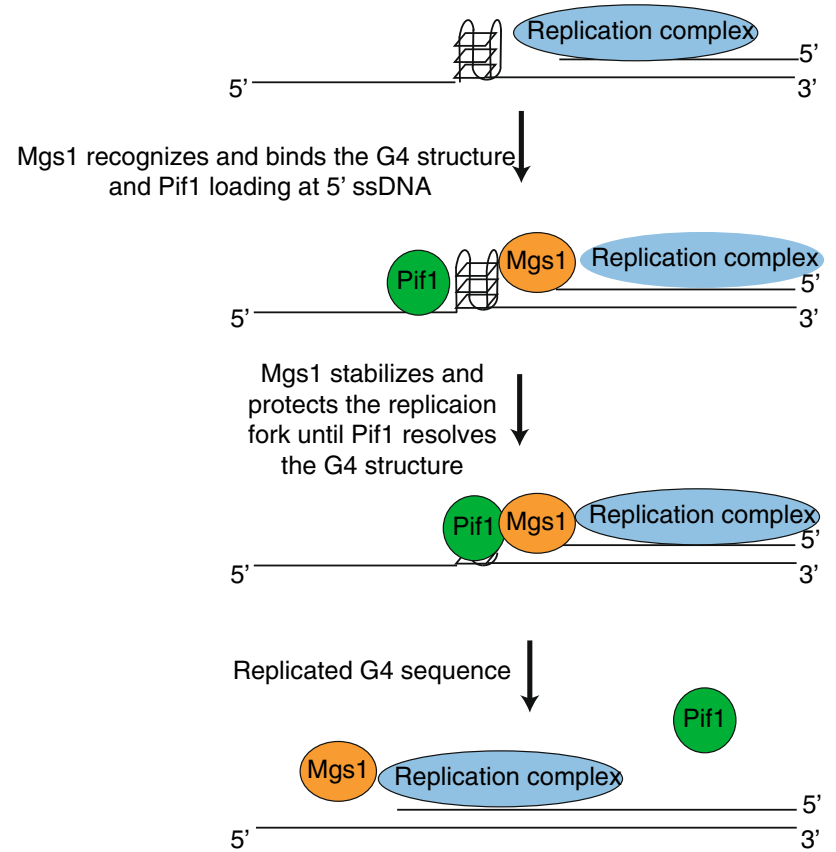

Fig. 1 Hypothetical model of the function of Mgs1 at G4 structures. During the replication G4 structures are formed on the single-stranded template DNA, which blocks the replication fork progression and DNA synthesis. Based on our results Mgs1 might be involved in the recognition of G4 structures at stalled replication forks. Binding of Mgs1 stabilizes and protects the replication fork until Pif1 resolves the blocking structure and joins the replication complex. This resumes the movement of the replication fork movement and replication beyond the G4 structure

G4 structure, the rescue of the stalled replication fork must depend on PCNA ubiquitylation; this process is not examined, yet. Answering this question could also bring us closer to understand the involvement of the different DNA polymerases in intrachromosomal G4 replication. However, the exact biochemical mechanism of Mgs1's function is still an open question. Identification of the amino acid residues of Mgs1 involved in G4-binding would be important to allow deeper analysis of Mgs 1 function at the G4 structure. Analysis of genetic interactions between $\mathrm{mgsl}$ and pif1 in non-G4 structure-associated pathways would also be important to understand their connection.

Acknowledgements This work was supported by National Research Development and Innovation Office [NKFIH K-119361] and Bolyai Janos Research Fellowship [to P.B.]. The work in the Paeschke lab is supported by an ERC Stg Grant (638988-G4DSB) and it is funded by the Deutsche Forschungsgemeinschaft (DFG, German Research Foundation) under Germany's Excellence Strategy - EXC2151 390873048. We thank Agnes Toth and Stefan Juranek for careful reading of the manuscript.
Funding Open access funding provided by ELKH Biological Research Center.

Open Access This article is licensed under a Creative Commons Attribution 4.0 International License, which permits use, sharing, adaptation, distribution and reproduction in any medium or format, as long as you give appropriate credit to the original author(s) and the source, provide a link to the Creative Commons licence, and indicate if changes were made. The images or other third party material in this article are included in the article's Creative Commons licence, unless indicated otherwise in a credit line to the material. If material is not included in the article's Creative Commons licence and your intended use is not permitted by statutory regulation or exceeds the permitted use, you will need to obtain permission directly from the copyright holder. To view a copy of this licence, visit http://creativecommons.org/licenses/by/4.0/.

\section{References}

Aguilera A, Garcia-Muse T (2013) Causes of genome instability. Annu Rev Genet 47:1-32

Arbel M, Liefshitz B et al (2020) How yeast cells deal with stalled replication forks. Curr Genet 66:911

Barbour L, Xiao W (2003) Regulation of alternative replication bypass pathways at stalled replication forks and its effects on genome stability: a yeast model. Mutat Res 532(1-2):137-155

Bochman ML, Paeschke K et al (2012) DNA secondary structures: stability and function of G-quadruplex structures. Nat Rev Genet 13(11):770-780

Bochman ML, Sabouri N et al (2010) Unwinding the functions of the Pif1 family helicases. DNA Repair (Amst) 9(3):237-249

Boule JB, Vega LR et al (2005) The yeast Pif1p helicase removes telomerase from telomeric DNA. Nature 438(7064):57-61

Branzei D, Seki M et al (2002a) The product of Saccharomyces cerevisiae WHIP/MGS1, a gene related to replication factor C genes, interacts functionally with DNA polymerase delta. Mol Genet Genomics 268(3):371-386

Branzei D, Seki M et al (2002b) Characterization of the slow-growth phenotype of S. cerevisiae Whip/Mgs1 Sgs1 double deletion mutants. DNA Repair (Amst) 1(8):671-682

Bryan TM (2019) Mechanisms of DNA replication and repair: insights from the study of G-quadruplexes. Molecules 24(19):3439

Budd ME, Reis CC et al (2006) Evidence suggesting that Pif1 helicase functions in DNA replication with the Dna2 helicase/nuclease and DNA polymerase delta. Mol Cell Biol 26(7):2490-2500

Burkovics P, Sebesta M et al (2013) Srs2 mediates PCNA-SUMOdependent inhibition of DNA repair synthesis. EMBO J 32(5):742-755

Buzovetsky O, Kwon Y et al (2017) Role of the Pif1-PCNA complex in pol delta-dependent strand displacement DNA synthesis and break-induced replication. Cell Rep 21(7):1707-1714

Byrd AK, Bell MR et al (2018) Pif1 helicase unfolding of G-quadruplex DNA is highly dependent on sequence and reaction conditions. J Biol Chem 293(46):17792-17802

Byrd AK, Raney KD (2015) A parallel quadruplex DNA is bound tightly but unfolded slowly by pif1 helicase. J Biol Chem 290(10):6482-6494

Capra JA, Paeschke K et al (2010) G-quadruplex DNA sequences are evolutionarily conserved and associated with distinct genomic features in Saccharomyces cerevisiae. PLoS Comput Biol 6(7):e1000861 
Chen Y, Yang D (2012) Sequence, stability, and structure of G-quadruplexes and their interactions with drugs. Curr Protoc Nucleic Acid Chem 50:17

Dahan D, Tsirkas I et al (2018) Pif1 is essential for efficient replisome progression through lagging strand G-quadruplex DNA secondary structures. Nucleic Acids Res 46(22):11847-11857

De Magis A, Gotz S et al (2020) Zuo1 supports G4 structure formation and directs repair toward nucleotide excision repair. Nat Commun 11(1):3907

Duan XL, Liu NN et al (2015) G-quadruplexes significantly stimulate Pif1 helicase-catalyzed duplex DNA unwinding. J Biol Chem 290(12):7722-7735

Foury F, Dyck EV (1985) A PIF-dependent recombinogenic signal in the mitochondrial DNA of yeast. EMBO J 4(13A):3525-3530

Galletto R, Tomko EJ (2013) Translocation of Saccharomyces cerevisiae Pif1 helicase monomers on single-stranded DNA. Nucleic Acids Res 41(8):4613-4627

Gotz S, Pandey S et al (2019) A novel G-quadruplex binding protein in yeast-Slx9. Molecules 24(9): 1774

Hansel-Hertsch R, Di Antonio M et al (2017) DNA G-quadruplexes in the human genome: detection, functions and therapeutic potential. Nat Rev Mol Cell Biol 18(5):279-284

Hishida T, Iwasaki H et al (2001) A yeast gene, MGS1, encoding a DNA-dependent $\mathrm{AAA}(+)$ ATPase is required to maintain genome stability. Proc Natl Acad Sci USA 98(15):8283-8289

Hishida T, Ohno T et al (2002) Saccharomyces cerevisiae MGS1 is essential in strains deficient in the RAD6-dependent DNA damage tolerance pathway. EMBO J 21(8):2019-2029

Hishida T, Ohya T et al (2006) Functional and physical interaction of yeast Mgs1 with PCNA: impact on RAD6-dependent DNA damage tolerance. Mol Cell Biol 26(14):5509-5517

Hoege C, Pfander B et al (2002) RAD6-dependent DNA repair is linked to modification of PCNA by ubiquitin and SUMO. Nature 419(6903):135-141

Hou XM, Wu WQ et al (2015) Molecular mechanism of G-quadruplex unwinding helicase: sequential and repetitive unfolding of G-quadruplex by Pif1 helicase. Biochem J 466(1):189-199

Huppert JL, Balasubramanian S (2007) G-quadruplexes in promoters throughout the human genome. Nucleic Acids Res 35(2):406-413

Ivessa AS, Zhou JQ et al (2000) The Saccharomyces Pif1p DNA helicase and the highly related Rrm3p have opposite effects on replication fork progression in ribosomal DNA. Cell 100(4):479-489

Jimenez-Martin A, Saugar I et al (2020) The Mgs1/WRNIP1 ATPase is required to prevent a recombination salvage pathway at damaged replication forks. Sci Adv 6(15):eaaz3327

Juranek SA, Paeschke K (2012) Cell cycle regulation of G-quadruplex DNA structures at telomeres. Curr Pharm Des 18(14):1867-1872

Kim JH, Kang YH et al (2005) In vivo and in vitro studies of Mgs1 suggest a link between genome instability and Okazaki fragment processing. Nucleic Acids Res 33(19):6137-6150

Lehmann CP, Jimenez-Martin A et al (2020) Prevention of unwanted recombination at damaged replication forks. Curr Genet 66:911

Lemmens B, van Schendel R et al (2015) Mutagenic consequences of a single G-quadruplex demonstrate mitotic inheritance of DNA replication fork barriers. Nat Commun 6:8909

Lerner LK, Sale JE (2019) Replication of G quadruplex DNA. Genes (Basel) 10(2):95

Li JR, Lu CY et al (2016) Multiple Pif1 helicases are required to sequentially disrupt G-quadruplex structure and unwind duplex DNA. Biochem Biophys Res Commun 473(4):1235-1239

Lipps HJ, Rhodes D (2009) G-quadruplex structures: in vivo evidence and function. Trends Cell Biol 19(8):414-422

Lopes J, Piazza A et al (2011) G-quadruplex-induced instability during leading-strand replication. EMBO J 30(19):4033-4046
Marsico G, Chambers VS et al (2019) Whole genome experimental maps of DNA G-quadruplexes in multiple species. Nucleic Acids Res 47(8):3862-3874

Mendoza O, Bourdoncle A et al (2016) G-quadruplexes and helicases. Nucleic Acids Res 44(5):1989-2006

Moldovan GL, Pfander B et al (2007) PCNA, the maestro of the replication fork. Cell 129(4):665-679

Motegi A, Kuntz K et al (2006) Regulation of gross chromosomal rearrangements by ubiquitin and SUMO ligases in Saccharomyces cerevisiae. Mol Cell Biol 26(4):1424-1433

Muellner J, Schmidt KH (2020) Yeast genome maintenance by the multifunctional PIF1 DNA helicase family. Genes (Basel) 11(2):224

Nakken S, Rognes T et al (2009) The disruptive positions in human G-quadruplex motifs are less polymorphic and more conserved than their neutral counterparts. Nucleic Acids Res 37(17):5749-5756

Paeschke K, Bochman ML et al (2013) Pif1 family helicases suppress genome instability at G-quadruplex motifs. Nature 497(7450):458-462

Paeschke K, Capra JA et al (2011) DNA replication through G-quadruplex motifs is promoted by the Saccharomyces cerevisiae Pif 1 DNA helicase. Cell 145(5):678-691

Papouli E, Chen S et al (2005) Crosstalk between SUMO and ubiquitin on PCNA is mediated by recruitment of the helicase Srs $2 p$. Mol Cell 19(1):123-133

Pfander B, Moldovan GL et al (2005) SUMO-modified PCNA recruits Srs 2 to prevent recombination during $\mathrm{S}$ phase. Nature 436(7049):428-433

Phillips JA, Chan A et al (2015) The pif1 helicase, a negative regulator of telomerase, acts preferentially at long telomeres. PLoS Genet 11(4):e1005186

Piazza A, Boule JB et al (2010) Genetic instability triggered by G-quadruplex interacting Phen-DC compounds in Saccharomyces cerevisiae. Nucleic Acids Res 38(13):4337-4348

Pike JE, Burgers PM et al (2009) Pif1 helicase lengthens some Okazaki fragment flaps necessitating Dna2 nuclease/helicase action in the two-nuclease processing pathway. J Biol Chem 284(37):25170-25180

Rhodes D, Lipps HJ (2015) G-quadruplexes and their regulatory roles in biology. Nucleic Acids Res 43(18):8627-8637

Ribeyre C, Lopes J et al (2009) The yeast Pif1 helicase prevents genomic instability caused by G-quadruplex-forming CEB 1 sequences in vivo. PLoS Genet 5(5):e1000475

Ripley BM, Gildenberg MS et al (2020) Control of DNA damage bypass by ubiquitylation of PCNA. Genes (Basel) 11(2):138

Rogers CM, Wang JC et al (2017) Yeast Hrq1 shares structural and functional homology with the disease-linked human RecQ4 helicase. Nucleic Acids Res 45(9):5217-5230

Sauer M, Paeschke K (2017) G-quadruplex unwinding helicases and their function in vivo. Biochem Soc Trans 45(5):1173-1182

Saugar I, Parker JL et al (2012) The genome maintenance factor Mgs1 is targeted to sites of replication stress by ubiquitylated PCNA. Nucleic Acids Res 40(1):245-257

Schulz VP, Zakian VA (1994) The saccharomyces PIF1 DNA helicase inhibits telomere elongation and de novo telomere formation. Cell 76(1):145-155

Sparks MA, Singh SP et al (2019) Complementary roles of Pif1 helicase and single stranded DNA binding proteins in stimulating DNA replication through G-quadruplexes. Nucleic Acids Res 47(16):8595-8605

Spiegel J, Adhikari S et al (2020) The structure and function of DNA G-quadruplexes. Trends Chem 2(2):123-136

Stith CM, Sterling J et al (2008) Flexibility of eukaryotic Okazaki fragment maturation through regulated strand displacement synthesis. J Biol Chem 283(49):34129-34140 
Sun H, Bennett RJ et al (1999) The Saccharomyces cerevisiae Sgs1 helicase efficiently unwinds G-G paired DNAs. Nucleic Acids Res 27(9):1978-1984

Todd AK, Neidle S (2011) Mapping the sequences of potential guanine quadruplex motifs. Nucleic Acids Res 39(12):4917-4927

Unk I, Hajdu I et al (2010) Role of yeast Rad5 and its human orthologs, HLTF and SHPRH in DNA damage tolerance. DNA Repair (Amst) 9(3):257-267

Valton AL, Prioleau MN (2016) G-quadruplexes in DNA replication: a problem or a necessity? Trends Genet 32(11):697-706

Varshney D, Spiegel J et al (2020) The regulation and functions of DNA and RNA G-quadruplexes. Nat Rev Mol Cell Biol 21(8):459-474

Vijeh Motlagh ND, Seki M et al (2006) Mgs1 and Rad18/Rad5/Mms2 are required for survival of Saccharomyces cerevisiae mutants with novel temperature/cold sensitive alleles of the DNA polymerase delta subunit, Pol31. DNA Repair (Amst) 5(12):1459-1474

Wagner M, Price G et al (2006) The absence of Top3 reveals an interaction between the Sgs1 and Pif1 DNA helicases in Saccharomyces cerevisiae. Genetics 174(2):555-573

Wanzek K, Schwindt E et al (2017) Mms1 binds to G-rich regions in Saccharomyces cerevisiae and influences replication and genome stability. Nucleic Acids Res 45(13):7796-7806
Wilson MA, Kwon Y et al (2013) Pif1 helicase and Poldelta promote recombination-coupled DNA synthesis via bubble migration. Nature 502(7471):393-396

Zacheja T, Toth A et al (2020) Mgs1 protein supports genome stability via recognition of G-quadruplex DNA structures. FASEB J 34:12646

Zhang B, Wu WQ et al (2016) G-quadruplex and G-rich sequence stimulate Pif1p-catalyzed downstream duplex DNA unwinding through reducing waiting time at ss/dsDNA junction. Nucleic Acids Res 44(17):8385-8394

Zhou R, Zhang J et al (2014) Periodic DNA patrolling underlies diverse functions of Pif1 on R-loops and G-rich DNA. Elife 3:e02190

Publisher's Note Springer Nature remains neutral with regard to jurisdictional claims in published maps and institutional affiliations. 